\title{
Voraussetzungen für eine erfolgreiche Implementierung
}

Für eine erfolgreiche Implementierung gilt, wie in allen Veränderungsprozessen, ein klares Commitment des Top-Managements. Ohne dessen Zuspruch kann ein solches Konzept nur schwerlich umgesetzt werden (Rosken 2016). Neben der reinen Entscheidungsnotwendigkeit für daran anschließende Maßnahmen, bedarf es auch Investitionen in gezielte Disability-Bereiche. Insofern ist die Zustimmung und der Support der Vorstandsebene bzw. des Senior Managements unabdingbar. Erfahrungen zeigen, dass eben jene Personen die Essentialität des Themas forcieren, welche selbst oder im privaten oder beruflichen Kontext von Disability betroffen sind. Die sich hier entwickelnde Empathie und das Verständnis führen zu einem klareren Verständnis und zur Unterstützung des Themas (Rosken 2020). Insofern sind Sensibilität und idealerweise erste Kenntnisse bzw. erstes Vorverständnis im Bereich des Dis-(Ability)-Managements von Vorteil. Beobachtungen aus der Praxis zeigen ferner, dass insbesondere Personen offen für das Thema sind, welche selbst von Disability „betroffen“ sind oder im unmittelbaren beruflichen oder privaten Kontext Menschen kennen, die eine Beeinträchtigung haben. Zum Beispiel, weil sie selbst erkrankt sind/waren, einen Angehörigen haben, der betreut werden muss oder Freunde haben, jene davon tangiert sind. Desweiteren kann die Professionalisierung des Disability Managements durch Zertifizierungslehrgänge zum Disability Manager zu mehr Erfolg führen. Zertifizierungen können zu einer Verstetigung des Disability Managements in Organisationen beitragen. Hier gibt es die Möglichkeit, Manager auszubilden oder auch ganze Betriebe zu klassifizieren. In Deutschland bietet federführend die Deutsche Gesetzliche Unfallversicherung Programme an. Es gibt aber auch andere Anbieter, welche ebenfalls Konzepte präsentieren. 